\title{
Enfermedad de Chagas en pacientes con miocardiopatía dilatada idiopática en Costa Rica
}

\author{
Oswaldo Gutiérrez-Sotelo', Silvia Molina-Solís ${ }^{2}$, Nidia Calvo-Fonseca ${ }^{3}$, Luis Romero-Triana ${ }^{4}$, \\ Lidieth Esquivel-Alfaro ${ }^{5}$, Erick Campos ${ }^{6}$
}

Resumen

Justificación y objetivo: Determinar la prevalencia de la enfermedad de Chagas en un grupo de pacientes con miocardiopatía dilatada, bradiarritmias y taquiarritmias ventriculares en Costa Rica.

Material y métodos: Estudio observacional, descriptivo, prospectivo de una serie de casos. Se seleccionaron pacientes con diagnóstico clínico y ecocardiográfico de miocardiopatía dilatada, bloqueo atrioventricular o intraventricular documentados o con arritmia ventricular compleja, excluyéndose aquellos en quienes hubiera uno o más factores causales (enfermedad arterial coronaria o cardiopatía hipertensiva). Mediante consentimiento informado, se les realizó un estudio con 2 pruebas serológicas de diferente fuente para detectar IgG anti-Trypanosoma cruzi.

Resultados: De aproximadamente 15.000 a 20.000 vistos pacientes por año en la Consulta Externa del Servicio de Cardiología del Hospital México, unos 500 se presentan con el diagnóstico de miocardiopatía dilatada. Excluidos los pacientes con causa conocida de su cardiopatía, entre febrero de 2004 y noviembre de 2005 se reclutó a 74 pacientes, 41 hombres y 33 mujeres, con edades comprendidas entre los 13 y los 87 años (promedio 47,9), de los cuales la mayoría ( $\mathrm{n}=51$;

1, 4, 5 Servicio de Cardiología, Hospital México

${ }^{2}$ Escuela de Medicina, Universidad de Costa Rica

3. ${ }^{6}$ Centro Nacional de

Referencia de Parasitología, INCIENSA

Abreviaturas: ELISA, ensayo inmunosorbente ligado a enzimas; $\operatorname{lgG}$, Inmunoglobulina $\quad \mathrm{G}$; INCIENSA, Instituto Costarricense de Investigación y Enseñanza en Nutrición y Salud

Correspondencia: Servicio de Cardiología, Sección 4-B, Hospital México e-mail: oswcr@hotmail.com oswcr@yahoo.com.ar

ISSN 0001-6002/2007/49/2/97-101 Acta Médica Costarricense, O2007 Colegio de Médicos y Cirujanos $68,9 \%$ ) tenían miocardiopatía dilatada idiopática, con una fracción de eyección promedio del $29,2 \% ; 18(24,3 \%)$ se incluyeron por bloqueo atrioventricular y 5 por arritmia ventricular compleja. De los 78 pacientes, $5(6,8 \%)$ resultaron tener serología positiva por enfermedad de Chagas; de estos: 3 con miocardiopatía dilatada, uno con bloqueo atrioventricular completo y uno con arritmia ventricular compleja. Cabe destacar que solo uno de los 3 pacientes dilatados tiene el electrocardiograma característico de la fase crónica de la enfermedad de Chagas: bloqueo AV de primer grado, bloqueo completo de rama derecha y hemibloqueo anterior izquierdo.

Conclusión: Los datos obtenidos en esta investigación revelan que la prevalencia de miocarditis chagásica crónica es relativamente baja entre los pacientes cardiópatas estudiados. Esta es la primera documentación clínica y serológica de la enfermedad en el país, en este grupo de pacientes. Entre las alteraciones clínicas predomina el daño miocárdico, pero también hay casos con bloqueo atrioventricular y arritmia ventricular compleja.

Descriptores: Arritmia ventricular compleja, bloqueo atrioventricular, miocardiopatía dilatada, miocarditis Chagásica

Key words: Chagasic myocarditis, dilated myocardiopathy, atrioventricular block, complex ventricular arrhythmia

Recibido: 31 de octubre de 2006

Aceptado: 6 de febrero de 2007 
La enfermedad de Chagas o tripanosomiasis americana es una parasitosis producida por la infección con el Trypanosoma cruzi, protozoario hemoflagelado que ingresa al ser humano a través de escoriaciones o por el contacto de la mucosa oral o conjuntiva ocular con las heces infectadas del Triatoma dimidiata, principal vector en nuestro país. ${ }^{1}$ Después del ingreso del parásito, se produce una infección aguda que cursa con fiebre, malestar y cefalea, por lo que con frecuencia es confundida con un cuadro gripal. Esta "fase aguda" se autolimita espontáneamente, alojándose el parásito en el sistema fagocítico mononuclear, sistema nervioso (especialmente plexos autonómicos y mioentéricos) $y$ en el miocardio. ${ }^{2}$ Desde este momento pueden pasar varios años y hasta décadas durante los cuales no hay manifestaciones clínicas (fase "indeterminada"), pudiendo sólo detectarse el contacto previo con el parásito mediante la determinación de anticuerpos contra diferentes antígenos del parásito.

Alrededor de un $30 \%$ de los pacientes infectados desarrollan luego de ese período, alteraciones autonómicas, megaesófago, megacolon y, especialmente, miocardiopatía dilatada, estadío denominado "fase crónica" de esta parasitosis. $^{2}$

La miocarditis chagásica crónica cursa con miocardiopatía dilatada con compromiso de 4 cámaras y aneurismas, en especial apicales, disfunción contráctil y por consiguiente insuficiencia cardiaca sistólica. Hay inflamación principalmente debida a la reacción inmune contra el parásito. ${ }^{3} \mathrm{El}$ daño en el sistema de conducción se caracteriza por disfunción sinusal con bradicardia significativa, paros sinusales y síndrome bradicardia-taquicardia. Hay también diversos grados de bloqueo atrioventricular y del sistema His-Purkinje. El bloqueo completo de la rama derecha del haz de His, más el bloqueo de la división anterior de la rama izquierda se han descrito como característicos de esta afección. ${ }^{4}$

El compromiso cardiaco autonómico se caracteriza por la presencia de hipotensión postural, fatiga $y$ el desencadenamiento de taquiarritmias ventriculares. Se atribuye a los anticuerpos antitripanosoma, la destrucción de los ganglios y nervios autonómicos cardiacos, predominando la disminución del tono parasimpático..$^{5-7}$

La arritmia ventricular es muy prevalente, al inicio se presenta como contracciones prematuras aisladas que se van haciendo cada vez más frecuentes y complejas, hasta llegar a la taquicardia ventricular monomorfa o polimorfa y muerte súbita cardiaca por fibrilación ventricular. ${ }^{8}$

En Costa Rica no se disponía de estimaciones nacionales sobre la prevalencia de la enfermedad de Chagas. Sin embargo, desde 2002. ${ }^{14,15}$ se han realizado diferentes tipos de estudios que la identifican como una enfermedad de baja prevalencia. ${ }^{9-15}$ Estudios epidemiológicos han revelado alta prevalencia de la infección en perros y otros portadores silvestres del parásito. ${ }^{16}$
Esta investigación tiene como objetivo conocer la prevalencia de la enfermedad de Chagas en pacientes con miocardiopatía dilatada de causa desconocida, atendidos en el Servicio de Cardiología del Hospital México.

\section{Materiales y métodos}

El protocolo de investigación fue aprobado por el Comité de Bioética de la Vicerrectoría de Investigación de la Universidad de Costa Rica y el Comité de Bioética del Instituto Costarricense de investigación y Enseñanza en Nutrición y Salud (INCIENSA).

Se realizó un estudio observacional, descriptivo, prospectivo, para determinar la prevalencia de la enfermedad de Chagas en pacientes atendidos en la consulta externa de cardiología del Hospital México. El estudio se inició en febrero de 2004 y se analizaron los casos incluidos hasta noviembre de 2005 .

Pacientes. Entre los 15.000 a 20.000 pacientes por año atendidos en la consulta del Servicio, se seleccionaron los individuos con diagnóstico clínico y ecocardiográfico de miocardiopatía dilatada, bloqueo atrioventricular o intraventricular de cualquier grado, o arritmia ventricular compleja.

Se excluyeron todos los pacientes que pudieran tener estos criterios, pero en quienes se conociera o documentara una causa específica atribuible a su miocardiopatía, como por ejemplo enfermedad arterial coronaria o cardiopatía hipertensiva, conocidas y muy frecuentes de estos trastornos miocárdicos.

A todos los pacientes se les realizó una valoración clínica, un electrocardiograma basal y un ecocardiograma de reposo. A discreción del médico tratante, se realizó prueba de esfuerzo, registro electrocardiográfico Holter y coronariografía, si el caso lo ameritaba, con el fin de documentar una causa atribuible a la cardiopatía encontrada. También se les solicitó el consentimiento informado por escrito.

Estudio serológico. A todos los pacientes seleccionados se les realizó una valoración con 2 pruebas serológicas de diferente fuente de antígenos, para detectar IgG antiTrypanosoma cruzi, ELISA de antígeno completo de T. cruzi y ELISA de una mezcla de antígenos recombinantes. En los casos con resultados discordantes, se utilizó una tercera prueba de inmunofluorescencia indirecta con cepas de epimastigotos costaricensis, por cuanto según los criterios de expertos de la Organización Mundial Salud, se cataloga a un paciente como chagásico cuando tiene 2 diferentes pruebas serológicas positivas. ${ }^{17}$ 
Los pacientes con resultado positivo fueron referidos al Servicio de Infectología para considerar tratamiento antiparasitario. Todos recibieron el tratamiento farmacológico convencional para la insuficiencia cardiaca, marcapasos para su bradiarritmia u otros tratamientos a discreción del médico tratante.

Análisis de los resultados. Se elaboró una base de datos; utilizando estadística descriptiva se caracterizó la población de estudio, y además se estableció la prevalencia de la infección chagásica y su relación con los hallazgos clínicos.

\section{Resultados}

Se seleccionaron 74 pacientes, 41 hombres y 33 mujeres, con edades entre 13 y 87 años (promedio 47,9). Solo un paciente se negó a participar en el estudio.

De los 74 pacientes incluidos, $51(68,9 \%)$ lo fueron por tener miocardiopatía dilatada idiopática con una fracción de eyección promedio del 29,2\%; dieciocho (24,3\%) se incluyeron por bloqueo atrioventricular y 5 por arritmia ventricular compleja.

Cinco pacientes tuvieron serología positiva para enfermedad de Chagas (Cuadro 1), resultando en una prevalencia del $6,8 \%$ en los pacientes con miocardiopatía dilatada de causa desconocida. De los 5 pacientes, 3 tienen miocardiopatía dilatada, uno bloqueo atrioventricular completo y uno arritmia ventricular compleja. Aunque ese fue el criterio de inclusión más evidente, en la mayoría se combinan los 3 en diverso grado.

Cabe anotar que solo uno de los 3 pacientes dilatados (y el único de todo el grupo) tiene el electrocardiograma característico de la fase crónica en la enfermedad, bloqueo atrioventricular de primer grado, bloqueo completo de rama derecha y hemibloqueo anterior izquierdo ${ }^{4}$ (Figura 1).

El paciente con arritmia ventricular compleja (número 58) presentó taquicardia ventricular incesante polimorfa, sin respuesta a ningún antiarrítmico solo ni en combinaciones. La ablación por catéter de la taquicardia fue fallida, por lo que se sometió a simpatectomía toracoscópica con importante mejoría electrocardiográfica y sintomática. Sin embargo, presentaba aún episodios de taquicardia ventricular no sostenida y falleció súbitamente a los 29 años, tras 4 meses, después del diagnóstico.

En relación con el lugar de procedencia, 2 pacientes son de Nicoya, 2 de Nicaragua y en uno éste no se pudo determinar. En los 4 de procedencia conocida, hubo condiciones de vivienda y ecológicas para el desarrollo del ciclo del parásito. No hubo ninguna otra diferencia clínica destacable con respecto al resto del grupo.

\section{Discusión}

Este estudio es el primer reporte que documenta miocarditis chagásica crónica en Costa Rica, en un grupo de pacientes portadores de miocardiopatía dilatada de causa desconocida. Si bien ha habido casos anecdóticos, no había datos que demostrasen su existencia de manera objetiva en esta población. Solo en años recientes se ha logrado instituir el tamizaje en bancos de sangre a nivel nacional en forma rutinaria, con lo cual se han detectado también pacientes seropositivos conocidos como sanos: solo algunos de estos tienen indicios de afectación cardiaca (INCIENSA, datos no publicados). Tal información permite afirmar que la

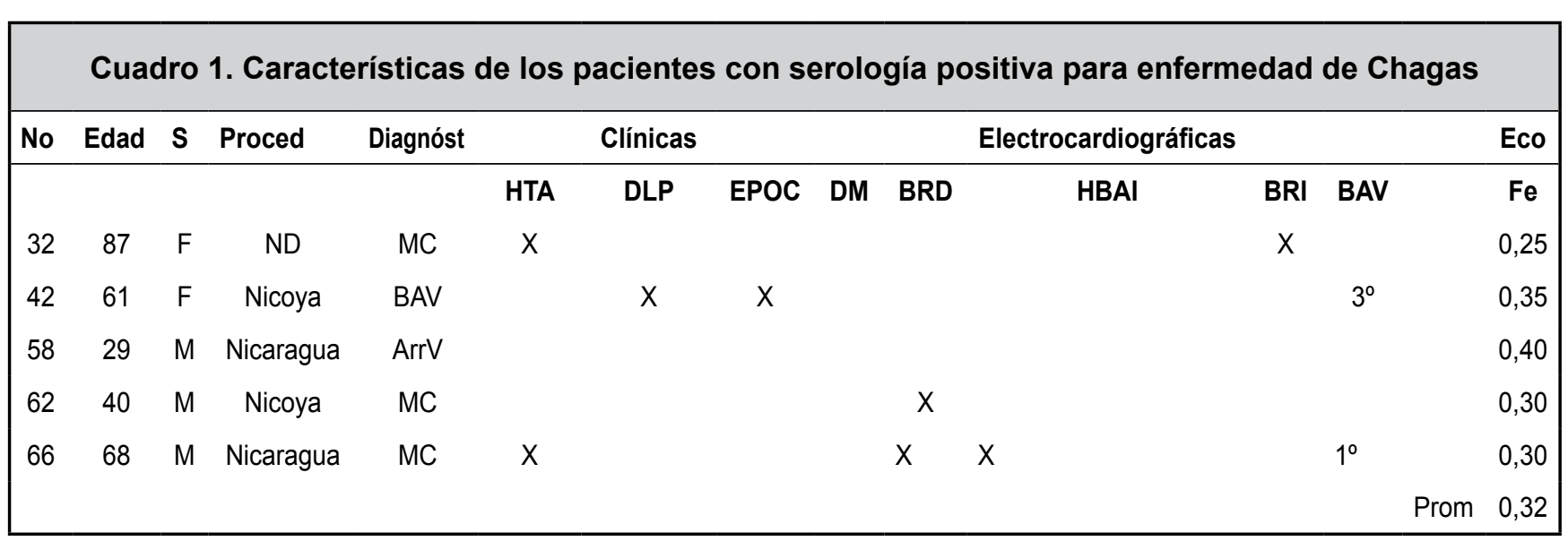

S, Sexo; Proced, Procedencia; CMD, miocardiopatía dilatada; BAV, bloqueo atrioventricular; ArrV, arritmia ventricular compleja; HTA, hipertensión arterial; DLP, dislipidemias; EPOC, enfermedad pulmonar obstructiva crónica; DM, diabetes mellitus; BRD, bloqueo de rama derecha; HBAl, hemibloqueo anterior izquierdo; ECO, ecocardiograma, Fe, fracción de eyección, ND, no disponible 


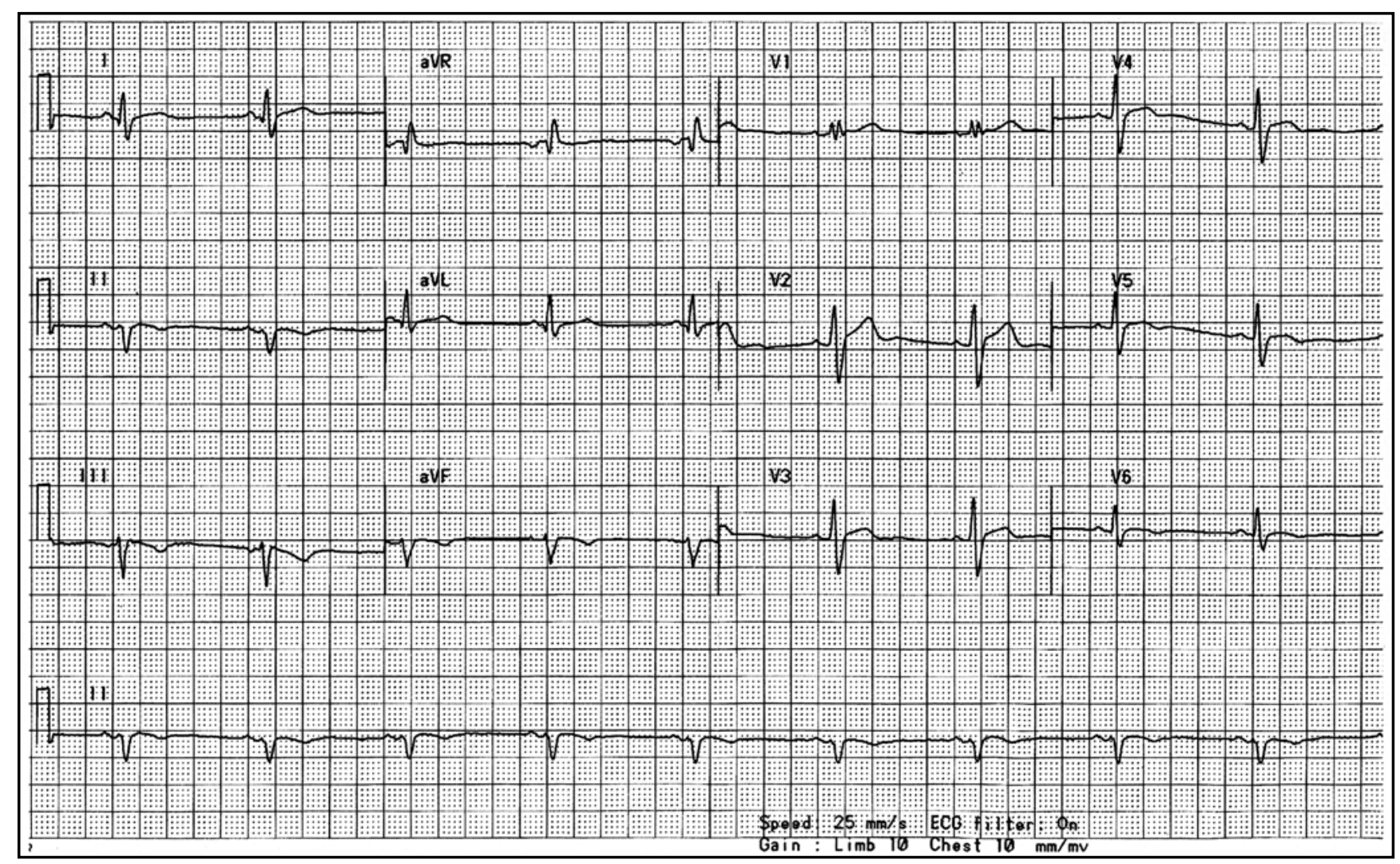

Figura. 1. Electrocardiograma del paciente No. 62 portador de miocardiopatía dilatada, quien resultó positivo para esta parasitosis. Se observa ritmo sinusal, bloqueo de rama derecha y hemibloqueo anterior izquierdo. El intervalo PR es normal en este caso.

enfermedad existe en el país más allá de algunos casos aislados $^{18}$ y que deben tomarse las medidas de prevención, detección y educación en la comunidad.

En el nivel latinoamericano se han señalado algunas diferencias regionales con respecto a la afectación del sistema de conducción, ${ }^{19}$ que se supone, se deben a la cepa de tripanosoma prevalente. De este estudio se infiere que las cepas presentes en el país producen tanto afectación miocárdica como del sistema de conducción.

Con respecto a la procedencia, si bien los 2 casos autóctonos son de Nicoya, en las encuestas serológicas y tamizajes en bancos de sangre se han diagnosticado personas provenientes de prácticamente todas las provincias del país. ${ }^{15}$

En este reporte hubo un caso de difícil manejo antiarrítmico, al que se le realizó estudio electrofisiológico con intento de ablación por catéter y terminó con simpatectomía toracoscópica. Si bien con este procedimiento mejoró sintomáticamente, finalmente falleció de súbito a los 29 años de edad. El caso es ilustrativo para destacar que, como en otras cardiopatías crónicas que afectan a individuos de un grupo de mayor edad, tal patología implica elevados costos para el sistema de salud e incapacidades laborales y muertes prematuras.
En conclusión, aunque la prevalencia de miocarditis chagásica crónica es baja entre pacientes cardiópatas, es la primera vez que se documenta clínica y serológicamente en el país en este grupo. Predomina la afectación miocárdica, pero también hay casos con bloqueo atrioventricular y arritmia ventricular compleja.

Dentro de las limitaciones del estudio se debe señalar que se realizó con la población adscrita a un centro hospitalario, por lo que no se pueden extrapolar los resultados en el nivel nacional. Hay un sesgo en el reclutamiento, puesto que no todos los pacientes portadores de miocardiopatía dilatada tienen acceso a la Consulta Externa del servicio, dado que son atendidos en otros centros.

\section{Financiamiento y aclaración}

- Este estudio fue realizado con financiamiento del proyecto de investigación $\mathrm{N}^{\circ}$ 422-A2-305, de la Vicerrectoría de Investigación de la Universidad de Costa Rica y la colaboración del Instituto Costarricense de Investigación y Enseñanza en Nutrición y Salud (INCIENSA).

- Este estudio fue presentado como resumen oral en el III Congreso Nacional de Cardiología. 


\section{Abstract}

Aim: To determine the prevalence of Chagas' disease in a group of patients from Costa Rica, with dilated myocardiopathy, bradiarrhythmias and ventricular tachyarrhythmias.

Materials and methods: We prospectively selected patients with clinical and echocardiographic diagnosis of dilated myocardiopathy, documented atrioventricular or intraventricular block or complex ventricular arrhythmias, excluding those who already had known etiologies, for example coronary artery disease or hypertensive heart disease. After obtaining an informed consent, we performed a serologic ELISA qualitative test using anti-trypanosoma cruzi antigens obtained by two different techniques.

Results: From aproximately 15,000 to 20,000 patients/year seen at the cardiology clinic of the Mexico Hospital, about 500 had dilated myocardiopathy. From February 2004 to august 2005 and excluding those patients with known causes, we recruited 74 patients, there were 41 men and 33 women with ages between 13 and 87 years (mean 47,8). Almost $70 \%(\mathrm{n}=51)$ were included because of idiopathic dilated myocardiopathy (mean ejection fraction 29,2\%), 24,3\% $(n=18)$ were included with atrioventricular block and 6,8\% $(n=5)$ with complex ventricular arrhythmia.

Five patients were positive, 3 with dilated myocardiopathy, 1 with atrioventricular blockade and 1 with complex ventricular arrhythmia. Of note, only 1 patient with dilated myocardiopathy (the only 1 in the group) had the charactersitic electrocardiographic pattern of the chronic phase of the disease: first degree atrioventricular block, right bundle branch block and left anterior division block. Antiarrhythmic management of the patient with complex ventricular arrhythmia (29 years old) was challenging. Since he did not respond to ablation and underwent sympathectomy, only to die 4 months later. There were no other differences compared with the other 69 patients.

Conclusion: The prevalence of chronic chagasic myocarditis is relatively low in patients with idiopathic myocardiopathy. This is the first time this subject is reported clinically and serologically in our country. In this entity myocardial compromise is predominant but there could also be atrioventricular blockade and complex ventricular arrhythmia cases.

\section{Referencias}

1. Starr MD, Rojas JC, Zeledón R, Hird DW, Carpenter TE. Chagas' disease: risk factors for house infestation by Triatoma dimidiata, the major vector of Trypanosoma cruzi in Costa Rica. Am J Epidemiol 1991; 133:740-7

2. Acquatella H, Mattia AR. A native of El Salvador with Tachycardia and Syncope. Case 32-1993. N Engl J Med 1993; 329:488-496

3. Golgher D, Gazzinelli RT. Innate and acquired immunity in the pathogenesis of Chagas disease. Autoimmunity 2004 5:399-409

4. Elizari MV, Chiale PA. Cardiac arrhythmias in Chagas' heart disease. J Cardiovasc Electrophysiol 1993 Oct; 4:596-608

5. Sterin-Borda L, Gorelik G, Borda ES. Chagasic IgG binding with cardiac muscarinic cholinergic receptors modifies cholinergicmediated cellular transmembrane signals. Clin Immunol Immunopathol 1991; 3:387-97

6. Goin JC, Perez Leiros C, Borda E, Sterin-Borda L. Modification of cholinergic-mediated cellular transmembrane signals by the interaction of human chagasic IgG with cardiac muscarinic receptors. Neuroimmunomodulation 1994; 5:284-91

7. Higuchi ML, Reis MM, Aiello VD, Benvenuti LA, Gutierrez PS, Bellotti G, Pileggi F. Association of an increase in CD8+ T cells with the presence of Trypanosoma cruzi antigens in chronic, human, chagasic myocarditis. Am J Trop Med Hyg 1997;56:485-489.

8. Manzullo EC, Chuit R Risk of death due to chronic chagasic cardiopathy. Mem Inst Oswaldo Cruz 1999; 94 Suppl 1:317-20

9. Visoná, K. Activity report for the LSU-ICMRT/ Red Cross Blood Bank Collaboration Program in Central America, 1995. San José, Costa Rica.

10. Martínez M, Taylor L, Visoná K. Prevalencia de anticuerpos antiChagas y anti-HTLV-1 en un grupo de donantes del Banco de Sangre del Hospital Nacional de Niños, Costa Rica. Rev Med Nal Niños (Costa Rica) 1994; 30:19-26.

11. Bonilla A, Moya T. Estudio serológico de la enfermedad de Chagas por la técnica de inmunofluorescencia indirecta. Trabajo final de graduación 1993 Facultad de Microbiología, Universidad de Costa Rica.

12. Zeledón R, Solano G, Burstin L, Swartzwelder J C. Epidemiological Pattern of Chagas Disease in a endemic area of Costa Rica. Am J Trop Med Hyg. 1975; 2;24: 214-225.

13. Chinchilla M, Montero-Gei F. Enfermedad de Chagas en Santa Ana - Costa Rica. Estudio parasicológico y serológico en 200 personas. Acta Méd Cost. 1968; 11; 211-217.

14. Calvo N, Zeledón R, Barboza MP, Sánchez G, Navas L. Encuesta serológica y entomológica para la enfermedad de Chagas en Costa Rica, 2001-2002. Boletín INCIENSA 2003; 15:3 Set- Dic

15. Sánchez G, Barboza MP, Calvo N. Conocimiento de la enfermedad de Chagas y probable forma de infección en donantes de sangre. Boletín INCIENSA 2004; 16:1.

16. Berrocal A, Morales JA, Cordero V, Villalobos C. Miocarditis aguda chagásica en caninos de Costa Rica. Cien Vet (Costa Rica) 1993; 15:51-59.

17. WHO. Control of Chagas Disease. Second Report of the WHO Expert Committee. WHO Bull 2002; No 905

18. Zeledón R, Solano G, Burstin L, Swartzwelder JC. Epidemiological pattern of Chagas' disease in an endemic area of Costa Rica. Am J Trop Med Hyg 1975; 24: 214-225.

19. Schofield CJ, Dias JC. The Southern Cone Initiative Against Chagas Disease. Adv Parasitol 1999;42:1-27 